\title{
INFLUENCIA DE LOS CAMBIOS CLIMÁTICOS EN LA ACUMULACIÓN DE CARBONO EN BOFEDALES ALTOANDINOS DURANTE LOS ÚLTIMOS 2500 AÑOS
}

\section{INFLUENCE OF CLIMATE CHANGES ON CARBON ACCUMULATION IN HIGH ANDEAN PEATLANDS DURING THE LAST 2500 YEARS}

\author{
Yizet Huaman ${ }^{1}$, Patricia Moreira-Turcq ${ }^{2}$, Raúl Espinoza ${ }^{3}$, Romina Llanos ${ }^{4}$, James \\ Apaéstegui ${ }^{5}$, Bruno Turcq ${ }^{6}$ y Bram Willems ${ }^{7}$
}

\begin{abstract}
Resumen
Las turberas alto andinas, llamadas también bofedales, presentan altas tasas de acumulación de carbono y sensibilidad a los cambios climáticos, motivo por el cual son consideradas como excelentes registros para estudios ambientales de alta resolución. El presente estudio reconstruye, a través del análisis del testigo APA01 ubicado en la cabecera de cuenca del río Cachi en Ayacucho, la historia paleoambiental de los últimos 2500 años. Las dataciones del testigo se determinaron por radiocarbono usando un Espectrómetro de Masas con Acelerador de Partículas (AMS), la estimación del carbono orgánico se realizó a través de un analizador elemental PDZ Europa ANCA-GSL. Los resultados evidencian cambios en las tasas de acumulación de carbono y en el clima local durante el Holoceno Tardío; específicamente durante los últimos 2500 años antes del presente. Durante el periodo denominado Anomalía Climática Medieval (ACM), alrededor de 1040 Después de Cristo (DC) a 1300 DC, el clima más seco provocó una desaceleración en la acumulación de carbono debido a una reducción del nivel freático que induce a una reducción en la producción de materia orgánica con un pico negativo en la concentración de carbono. Al final de la Pequeña Edad de Hielo (PEH), entre 1600 DC y 1890 DC, la acumulación de carbono presentó alta variabilidad. Posteriormente, el ambiente de turba se caracteriza principalmente por un aumento considerable de las tasas de acumulación de carbono debido al aumento de la tasa de sedimentación que posiblemente esté relacionado con el retroceso de los glaciares en los Andes centrales. El reciente aumento drástico en la tasa de sedimentación y la reducción de las concentraciones de carbono advierten de una posible disminución futura de estos ecosistemas.
\end{abstract}

Palabras clave: Andes, carbono, tasas de acumulación, bofedal, paleoclima.

\begin{abstract}
High-altitude cushion peatlands contain important records for high-resolution palaeoenvironmental studies, due to their high carbon accumulation rates and sensitivity to climatic changes. In this study, we present the paleoenvironmental history for the last 2500 years based on the study of APA01 core, located in the headwaters of the Cachi river basin in Ayacucho. Radiocarbon dating by an accelerator mass spectroscopy (AMS) and by an elemental PDZ Europa ANCA-GSL analyzer to estimate particulated organic carbon were determined. Our results reveal changes in the peatlands during the Late Holocene. The palaeo climate varied significantly during the last 2500 years. During the Medieval Climatic Anomaly (ACM) from around 1040 DC (Before Christ - BC, in spanish paper) to $1300 \mathrm{DC}$, the drier climate caused a slowdown in peat accumulation due to a reduced water table inducing a reduced organic matter production with a negative peak of carbon concentration. At the end of the Small Ice Age (PEH), between 1600 DC and 1890 DC, carbon accumulation showed high variability. Subsequently, the peat environment is mainly characterized by a considerable increase of carbon accumulation rates due to high sedimentation rates that is possibly related to the retreat of glaciers in the Central Andes. The recent drastic increase in the sedimentation rate and the reduction of carbon concentrations are a warning of a possible future decline of these ecosystems.
\end{abstract}

Key words: Andes, carbon, accumulation rates, peatlands, paleoclimate.

\section{Introducción}

Las turberas contienen un tercio del carbono (C) orgánico global del suelo, cubriendo solo del 4 a $5 \%$ de la superficie terrestre, y son un componente importante del ciclo del $\mathrm{C}$ a nivel mundial (Waddington et al., 2003). Las turberas altoandinas, llamadas bofedales, son caracterizadas por la presencia de plantas en forma 

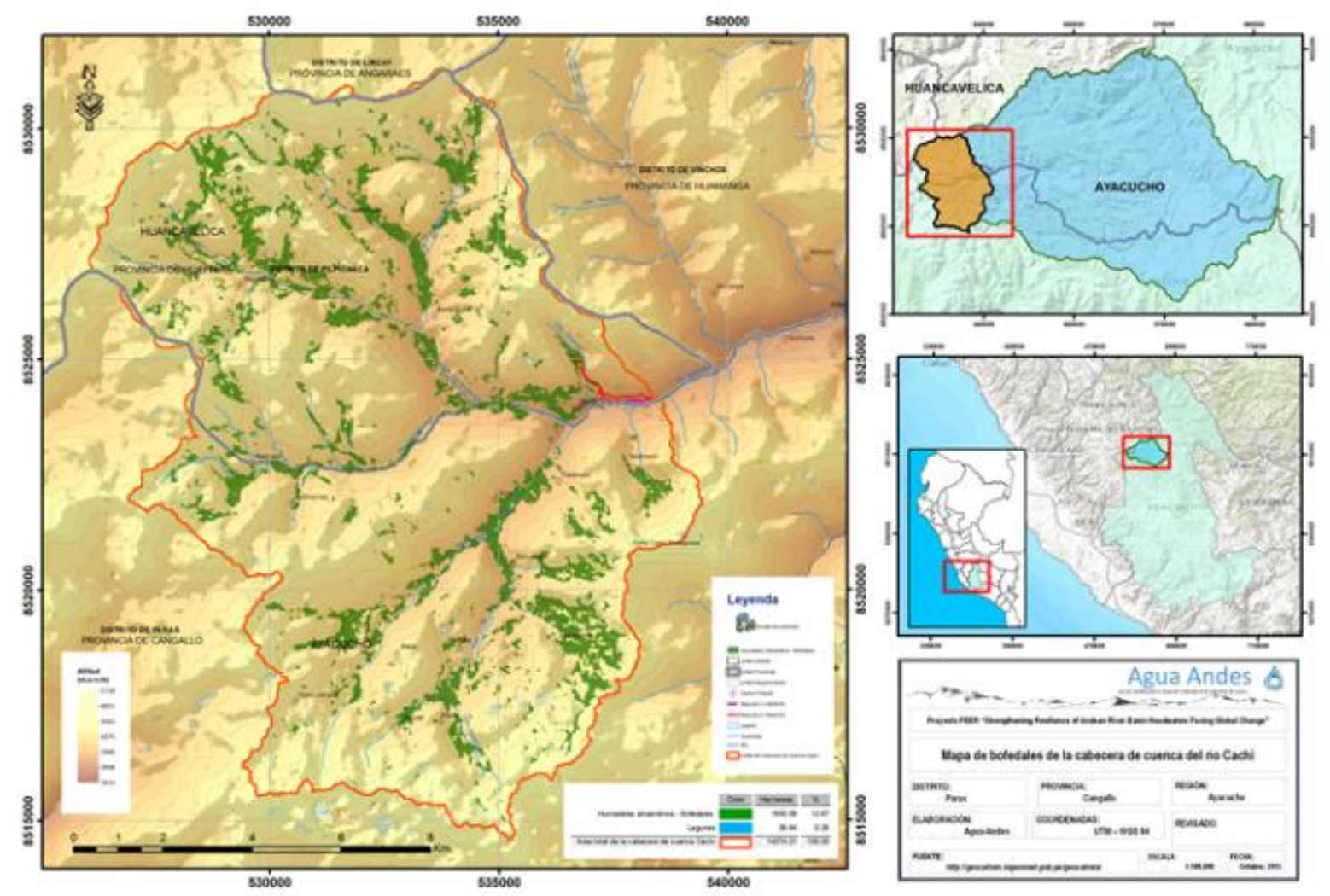

Figura 1. Localización de la región de estudio con la cabecera de la cuenca del río Cachi donde fue colectado el testigo APA01.

de cojines y son muy sensibles a los cambios climáticos que afectan, entre otros, sus regímenes hidrológicos, las condiciones redox y la geoquímica local (Schittek et al., 2016). Estos sistemas, característicos de los Andes, son poco explorados, a pesar de representar excelentes registros para estudios paleoclimáticos de alta resolución (Schittek et al., 2015).

Cambios climáticos (variaciones de temperatura y/o precipitación) a escala global durante el Holoceno Tardío, periodo correspondiente a los últimos 4000 años antes del presente, indujeron respuestas significativas (avance y retroceso de los glaciares) en la Cordillera de los Andes centrales (Thompson et al., 1994; Thompson, 1995). Las turberas desempeñaron también un papel importante en el ciclo global del C durante el Holoceno Tardío, como lo reconoció el informe del IPCC (2013); sin embargo, todavía no comprendemos la sensibilidad de estos ecosistemas frente al cambio climático, especialmente a escala continental y regional.

Comprender la sensibilidad climática y la contribución de los depósitos de turba al ciclo global del $\mathrm{C}$ en el pasado, y también su influenza en las concentraciones de $\mathrm{CO}_{2}$ y $\mathrm{CH}_{4}$ en la atmósfera, es fundamental para proyectar su cambio en el futuro. La mayoría de los estudios acerca de la captura del $\mathrm{C}$ en los suelos en Perú se han enfocado en bosques y cultivos, a pesar de la importancia de los bofedales. El clima es el factor de control más importante de la distribución y extensión de las turberas a nivel mundial; en consecuencia, cualquier cambio climático futuro tendrá un impacto en su distribución y/o declino.

El objetivo de este trabajo fue evaluar el rol de las turberas altoandinas en la acumulación de $\mathrm{C}$ a lo largo de los últimos 2500 años AP (Antes del Presente) y comprender de qué manera los cambios climáticos pasados impactaron estos sistemas.

\section{Área de estudio}

El área de estudio se localiza en un bofedal de la quebrada del río Apacheta de la cabecera de cuenca del río Cachi, en la Cordillera Central de los Andes Peruanos a $4210 \mathrm{msnm}$ donde se recolectó el testigo APA01 en la coordenada $13^{\circ} 21^{\prime} 4.61$ ', latitud sur y $74^{\circ} 39^{\prime} 31.75^{\prime}$ ' longitud oeste (Figura 1). El río Cachi es la principal fuente de recursos hídricos para el abastecimiento de la ciudad de Huamanga, capital de la provincia de Ayacucho.

Geológicamente, la cabecera de cuenca del río Cachi está situada en un área donde predomina la Andesita, que es una roca ígnea volcánica, y que corresponde a una zona de transición entre Puna húmeda y seca. 
La precipitación en Sudamérica exhibe una marcada asimetría zonal, con condiciones muy húmedas (secas) al oeste (este) de la Cordillera de los Andes (Garreaud et al., 2009). Por lo que, en nuestra zona de estudio (Ayacucho) la precipitación proviene de las masas de aire húmedas del Océano Atlántico, que son transportadas hacia la cordillera de los Andes por el flujo oriental del nivel superior y esta a su vez es controlada en parte por el Fenómeno El NiñoOscilación del Sur (ENOS).

Cerca del 90\% del total de lluvia está concentrada durante los meses del verano austral que comprende desde Noviembre hasta Marzo (Garreaud, 2000). Esta variabilidad estacional de las lluvias está conectada con la ocurrencia del monzón sudamericano (Zhou \& Lau, 1998), donde parte del vapor reciclado de la Amazonia es transportado hacia el sur-este de los Andes. Los veranos son cortos, húmedos y nublados, mientras que los inviernos también son cortos, secos y parcialmente nublados. Se le conoce como un clima de estepa local. De acuerdo con Köppen y Geiger, el clima se clasifica como BSk. Durante el año la temperatura varía de $8{ }^{\circ} \mathrm{C}$ a $22{ }^{\circ} \mathrm{C}$ y en ocasiones baja a menos de $5{ }^{\circ} \mathrm{C}$, con una media anual de $15.4{ }^{\circ} \mathrm{C}$ y precipitaciones promedio de $575 \mathrm{~mm}$

En la cabecera de cuenca del río Cachi existen varias fuentes de agua que alimentan la turba minerotrófica del bofedal. El área de acumulación de turba se ve desde la parte superior del bofedal hasta su confluencia con el río tributario Apacheta (Figura 1). Los "cojines" que se encuentran en los bofedales a altas altitudes se encuentran a lo largo de la cordillera de los Andes con composición florística gradual cambiante (Squeo et al., 2006; Ruthsatz, 2008). Para el caso de la cabecera de cuenca del río Cachi, la Juncaceae Distichia muscoides es la planta predominante para la formación de cojines de turba en los bofedales. A menudo crecen tan densamente que pueden formar extensas alfombras, estables, que varían en forma desde casi planas a semiesféricas. Los brotes siguen creciendo en sus copas pero van muriendo en el fondo (Rauh, 1998).

\section{Metodología}

El trabajo de campo se llevó a cabo en abril del 2015. Para elegir el punto de ubicación del testigo APA 01 (Figura 1) se realizó un mapeo de los bofedales en la cabecera de cuenca a diferentes altitudes. El testigo fue recolectado manualmente en un tubo de PVC con un diámetro de 3 pulgadas, con una profundidad de 96 $\mathrm{cm}$. Este fue abierto longitudinalmente en dos partes iguales utilizando una sierra circular. Después de la abertura, el testigo fue inmediatamente radiografiado (con un SCOPIX de la Universidad de Bordeaux, Francia) y descrito para evitar la alteración del sedimento por la oxidación y, al mismo tiempo, fue descrito según sus características sedimentológicas, macroscópicas (textura) y ópticas (color) con la ayuda de una tabla de colores de Munsell. Las muestras para las dataciones con carbono $14\left({ }^{14} \mathrm{C}\right)$ fueron escogidas de acuerdo con las variaciones de textura y color que normalmente indican cambios en la sedimentación.

Para determinar la edad de los depósitos sedimentares, las muestras seleccionadas fueron secadas en un horno a $50{ }^{\circ} \mathrm{C}$ por algunos días hasta obtener peso constante (Andrejko et al., 1983). Seguido, las muestras fueron enviadas al Laboratorio de Medida de Carbono 14 (LMC14-Francia), donde fueron analizadas en un Espectrómetro de Masas con Acelerador de Partículas (AMS) para la estimación del isótopo ${ }^{14} \mathrm{C}$.

Las edades presentadas en este trabajo fueron debidamente calibradas, los rangos de edad calibrados se calcularon utilizando CALIB 7.10 (programa Calib 7.10 disponible en internet (http://calib.org/calib/calib.html) e Intel Cal 13.14c (Hua et al., 2013; Reimer et al., 2013). El modelo cronológico fue obtenido con el uso del paquete Clam 2.2 en el software R. Las edades obtenidas fueron expresadas en años AC y DC (Antes de Cristo y Después de Cristo, respectivamente).

Se fraccionó una de las mitades de estos testigos, con una separación de un centímetro entre cada corte, y se secaron las muestras resultantes en una estufa a una temperatura de $50{ }^{\circ} \mathrm{C}$. Con los datos de volumen húmedo y peso seco de esta parte del procedimiento se obtuvo la densidad aparente $\left(\mathrm{g} . \mathrm{cm}^{-3}\right)$. El análisis de carbono orgánico total (COT) fue realizado en un analizador elemental PDZ Europa ANCA-GSL.

La tasa de acumulación de carbono (TAC), o flujo de carbono, fue calculado a través de la multiplicación de la concentración de COT por la densidad aparente de cada muestra y éste resultado fue multiplicado por la tasa de sedimentación $\left(\mathrm{cm} \cdot \mathrm{año}^{-1}\right)$ obtenido del modelo cronológico; de esta manera la TAC es expresada en $\mathrm{g} \cdot \mathrm{m}^{-2} \cdot \mathrm{año}^{-1}$, representada por la Ecuación 1 :

$$
\mathrm{TAC}=\operatorname{COT} \times \mathrm{D}_{\mathrm{a}} \times \mathrm{TS} \ldots(\text { Ecuación } \mathbf{1})
$$

Donde:

TAC :Tasa de acumulación de carbono $\left(\mathrm{g} \cdot \mathrm{m}^{-2} \cdot \mathrm{año}^{-1}\right)$; COT :Carbono orgánico total (\%);

Da :Densidad aparente $\left(\mathrm{g} \cdot \mathrm{cm}^{-3}\right)$; y

TS :Tasa de sedimentación $\left(\mathrm{cm} \cdot \mathrm{añno}^{-1}\right)$.

\section{Resultados y discusión}

\section{Descripción litológica y cronología}

La descripción litológica del testigo APA01, de acuerdo con su textura, características de color y sedimentológicas, reflejan una dominancia de arcilla orgánica y restos vegetales. Los restos vegetales disminuyen gradualmente con la profundidad mientras que la arcilla orgánica aumenta.

Nueve dataciones radiocarbono fueron realizadas para este estudio; los resultados de las edades 
(calibradas y no calibradas) son presentados en la Tabla 1.

El modelo cronológico edad-profundidad fue hecho con todas las dataciones radiocarbono. El testigo APA 01 presenta una edad aproximada de 2500 años. La curva escogida para el modelo cronológico fue "spline suavizado" (Figura 2) que permite observar dos patrones de sedimentación muy pronunciados a lo largo de los últimos 2500 años calibrados AC/DC. Las tasas de sedimentación fueran calculadas a partir de este modelo de sedimentación.

A través de la cronología establecida del testigo APA01 fue posible identificar variaciones en las tasas de sedimentación (más lentas o más rápidas) a lo largo de los últimos 2500 años, ésta a su vez fue dividida en dos unidades sedimentarias (la Unidad 1 y 2). Un periodo (Unidad 2, Tabla 2) caracterizado por una tasa de sedimentación constante y relativamente lenta $\left(0.027 \mathrm{~cm} \cdot a_{\tilde{n}}{ }^{-1}\right)$ entre la base del testigo hasta el año $1600 \mathrm{DC}$, a $38 \mathrm{~cm}$, que corresponde al comienzo de la Pequeña Edad del Hielo (PEH); y un segundo periodo (Unidad 1, Tabla 2) hasta la superficie donde la tasa de sedimentación es muy rápida $\left(0.251 \mathrm{~cm} \cdot \mathrm{año}^{-1}\right)$, con una pendiente muy pronunciada a partir de 1975, aproximadamente. También fue posible identificar algunos de los principales periodos de cambios climáticos a lo largo del Holoceno Tardío, como por ejemplo, la Anomalía Climática Medieval (ACM), entre el año 1000 y 1350 DC, y la Pequeña Edad del Hielo (PEH), entre el año 1600 y 1890 DC.

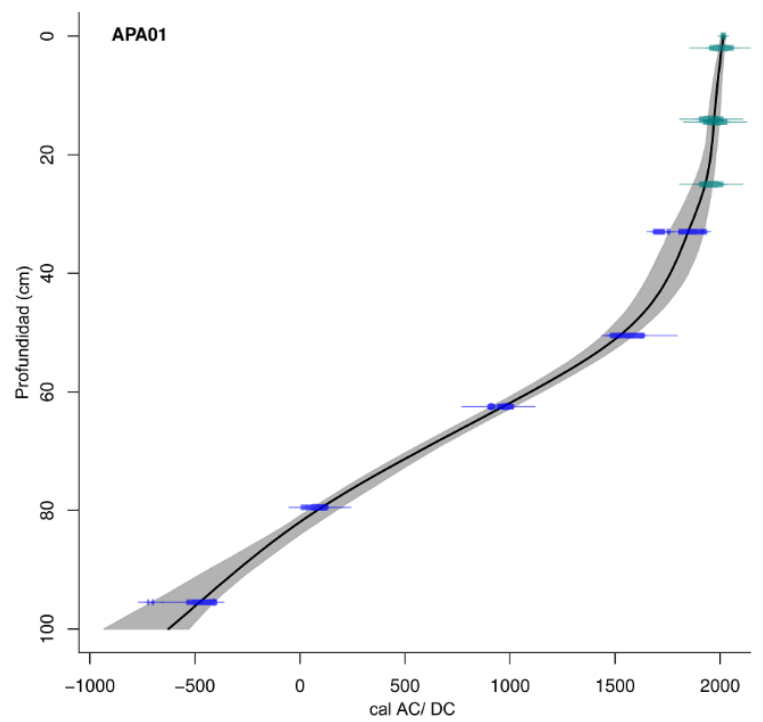

Figura 2. Modelo cronológico expresado en profundidad (cm) vs edad (años) para el testigo APA01 durante los últimos 2500 años en base a nueve dataciones ${ }^{14} \mathrm{C}$.

\section{Variabilidad geoquímica del testigo}

El análisis de la densidad, del material orgánico y de las tasas de acumulación de C del testigo APA01, nos permite observar variaciones marcadas a lo largo de los últimos 2500 años (Figura 3).

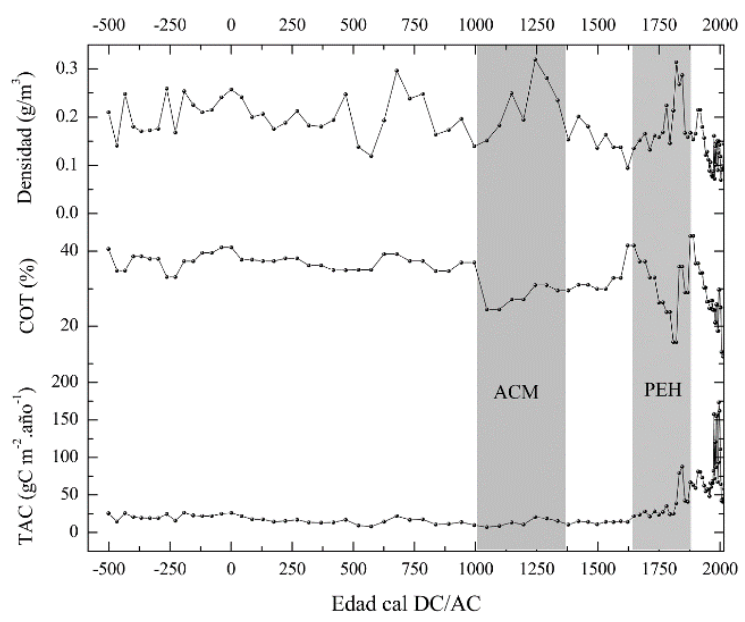

Figura 3. Variabilidad de la densidad (muy poroso), $\%$ carbono y la TAC a lo largo de 2500 años, en gris periodos de ACM (Anomalía Climática Medieval, periodo más seco) y la PEH (Pequeña Edad de Hielo, periodo más frio).

De acuerdo al modelo cronológico (Figura 2), se dividió el testigo en dos unidades sedimentarias: la Unidad 2, de 96 a $38 \mathrm{~cm}$, y la Unidad 1, de $38 \mathrm{~cm}$ hasta la superficie. En la Tabla 2 se muestran los valores máximos y mínimos de densidad, carbono orgánico total, tasas de sedimentación y tasas de acumulación de carbono para cada unidad sedimentaria. Hasta el año 1600 DC no observamos muchas variaciones en la acumulación de material orgánico ni de la densidad. La tasa de acumulación también es relativamente estable. Con el aumento de la tasa de sedimentación, a partir de $1600 \mathrm{AC}$, es que tenemos un rápido aumento de acumulación de material orgánico. En este estudio observamos que a lo largo de los últimos 2500 años las tasas de acumulación de carbono variaron considerablemente (Figura 3), con tres periodos bien distintos (Figura 4). Un periodo de pequeñas variaciones que va de $515 \mathrm{AC}$ hasta $1600 \mathrm{DC}$, donde tenemos la ACM y donde la media de la tasa de acumulación de carbono es de $16.7 \mathrm{~g} \cdot \mathrm{m}^{-2} \cdot \mathrm{año}^{-1}$; un segundo periodo durante la $\mathrm{PEH}$, donde observamos un aumento gradual durante 300 años, con algunos picos, y caracterizado por una tasa promedio de $41 \mathrm{~g} \cdot \mathrm{m}^{-2} \cdot \mathrm{año}^{-}$ ${ }^{1}$ de C.

Finalmente, a partir de los últimos 100 años, la variabilidad es notoria, con una media de $85 \mathrm{~g} \cdot \mathrm{m}^{-2}$. año${ }^{1}$ de $\mathrm{C}$ y picos máximos de hasta $173 \mathrm{~g} \cdot \mathrm{m}^{-2} \cdot \mathrm{año}^{-1} \mathrm{de} \mathrm{C}$ (Figura 4). Una de las principales causas de estas variaciones está relacionada a los cambios de las tasas de sedimentación (Tabla 2).

En cuanto a las concentraciones de $\mathrm{C}$, disminuyen durante la ACM (Figuras 3 y 4) y la tasa de acumulación en este periodo no muestra alteración, 
siendo casi constante (Unidad 2), pudiendo ser un período más seco y prolongado (Apaéstegui et al., 2014). Este periodo corresponde a una fase climática más seca durante la ACM descrita por Apaéstegui et al. (2014). Este clima seco puede ser responsable de la reducción de la producción vegetal y/o el aumento de la degradación de la materia orgánica. Esta sequía culminó durante 1040-1100 DC, que es el periodo más seco de la ACM (Apaéstegui et al., 2014). Sin embargo, durante la $\mathrm{PEH}$, el \% COT varía considerablemente y la TAC deja de ser constante, presentando un pico muy resaltante alrededor del año 1845 (Unidad 1). Por esta transición, entre la Unidad 2 y 1 , y considerando las concentraciones de $\mathrm{C}$, podemos intuir que este periodo fue mixto (Frio/Seco y Frio/Húmedo). Respecto a los últimos 100 años, el C demuestra una clara tendencia a disminuir (Figura 4) desde 1900 DC. Sin embargo, observamos las mayores tasas de sedimentación y de acumulación de carbono, que son encontradas después de los 70's $\left(97 \mathrm{~g} \cdot \mathrm{m}^{-2} \cdot\right.$ año $\left.^{-1}\right)$. Estos resultados muestran claramente que las altas tasas de acumulación de carbono no están directamente relacionadas, en este periodo, con las concentraciones de carbono, porque estas disminuyeron de 35\% en el año 1900 DC hasta un $10 \%$ en el 2015, pero sí con las tasas de sedimentación. Esto nos lleva a pensar que este aumento de la tasa de acumulación de carbono es producto, principalmente, de un gran aporte de material mineral, responsable de un aumento de las tasas de sedimentación, que podría estar relacionado al retroceso de los glaciares y que debe su máxima aceleración en los años 70's (Rabatel et al., 2013).

Las altas tasas de acumulación de carbono encontradas en el bofedal están también relacionadas al tipo de vegetación de la turba, dominada en la mayor parte de los humedales alto andinos del Perú por la especie Distichia muscoides, cuyas plantas crecen formando una suerte de cojines (Schittek et al., 2015). Las plantas en forma de cojín son responsables de una alta acumulación de materia orgánica, promoviendo una alta diversidad local, así como, también, funcionan como reguladores hídricos, reteniendo agua sin sobresaturación.

\section{Conclusión}

Este estudio muestra que la acumulación de carbono a lo largo del tiempo no fue constante en los bofedales Andinos debido a que los últimos 2500 años estuvieron marcados por periodos de muy fuerte acumulación de carbono y otros de menor acumulación debido a las alteraciones del clima. Sin embargo, en los últimos 50 años los bofedales parecen estar en condiciones muy sensibles a los cambios climáticos regionales que influyen su tasa de sedimentación y las características de esta sedimentación.

$\mathrm{El}$ análisis de la concentración de $\mathrm{C}$ en el testigo sedimentario pone en evidencia que los bofedales son sensibles a los cambios del clima, puesto que la concentración de $\mathrm{C}$ disminuye levemente en la ACM (periodo más caliente pero con glaciar permanente), $\mathrm{y}$ la variabilidad del COT en la PEH es muy alterada en este periodo, debido a diversos avances y retrocesos en los glaciares. El avance de los glaciares en la región fue relacionado con la disminución de las tasas de acumulación de material orgánico y viceversa. Actualmente, el drástico aumento de la tasa de sedimentación y la diminución de las concentraciones de carbono alerta un posible declino futuro de estos ecosistemas.

\section{Agradecimientos}

Esta investigación se desarrolló gracias al soporte financiero del programa USAID - Partnership for Enhanced Engagement in Research (PEER) a través de los proyectos: "Strengthening resilience of Andean river basin headwaters facing global change" (PGA084063) y "AGUA-ANDES: Ecological Infrastructure Strategies for Enhancing Water Sustainability in the Semi-Arid Andes" (PGA-174194); y gracias a la Red Internacional de Seguridad del Agua (IWSN) financiada por la Fundación Lloyd's Register. Y del IRD (Institut de recherche pour le développement de Francia). Esta investigación también recibió el apoyo financiero del Proyecto MAGNET "Clima, paleoambientes y biodiversidad en sistemas marinos y acuáticos continentales" - Subproyecto N4 "Sensibilidad de los ecosistemas acuáticos de montaña frente a los cambios climáticos" del CONCYTEC, Perú.

\section{Literatura citada}

Andrejko M., Fiene F., \& Cohen A. 1983. Comparison of Ashing Techniques for Determination of the Inorganic Content of Peats. In: Jarrett, P. (ed.) STP820-EB Testing of Peats and Organic Soils, West Conshohocken. PA: ASTM International. DOI: https://doi.org/10.1520/STP37331S. 5-20.

Apaéstegui J., Cruz F.W., Sifeddine A., Vuille M., Espinoza J.C., Guyot J.L., Khodri M., Strikis N., Santos R.V., Cheng H., Edwards L., Carvalho E. \& Santini W. 2014. Hydroclimate variability of the northwestern Amazon Basin near the Andean foothills of Peru related to the South American Monsoon System during the last 1600 years. Climate of the Past, 10(6): 1967-1981. DOI: 10.5194/cp-10-1967-2014.

Garreaud R. 2000. Intraseasonal Variability of Moisture and Rainfall over the South American Altiplano. Monthly Weather Review, 128(9): 3337-3346. DOI: $10.1175 / 1520-$ 0493(2000)128<3337:IVOMAR>2.0.CO;2.

Garreaud R.D., Vuille M., Compagnucci R. \& Marengo J. 2009. Present-day South American climate. Palaeogeography, Palaeoclimatology, Palaeoecology, 281(3-4): 180-195. DOI: 10.1016/j.palaeo.2007.10.032.

Hua Q., Barbetti M. \& Rakowski A.Z. 2013. Atmospheric Radiocarbon for the Period 1950-2010. Radiocarbon, 55(04): 2059-2072.

10.2458/azu_js_rc.v55i2.16177. 
IPCC. 2013. Contribución del grupo de trabajo 1 al quinto informe de evaluación del grupo intergubernamental de expertos sobre el cambio climático.

Rabatel A., Francou B., Soruco A., Gomez J., Cáceres B., Ceballos J.L., Basantes R., Vuille M., Sicart J.-E., Huggel C., Scheel M., Lejeune Y., Arnaud Y., Collet M., Condom T., Consoli G., Favier V., Jomelli V., Galarraga R., Ginot P., Maisincho L., Mendoza J., Ménégoz M., Ramirez E., Ribstein P., Suarez W., Villacis M. \& Wagnon P. 2013. Current state of glaciers in the tropical Andes: A multi-century perspective on glacier evolution and climate change. Cryosphere, 7(1): 81-102. DOI: 10.5194/tc-7-81-2013.

Rauh W. 1998. Tropische Hochgebirgspflanzen: Wuchs-und Lebensformen. Nordic Journal of Botany, 14(4): 88192. Springer-Verlag.

Reimer P.J., Bard E., Bayliss A., Beck J.W., Blackwell P.G., Bronk Ramsey C., Buck C.E., Cheng H., Edwards R.L., Friedrich M., Grootes P.M., Guilderson T.P., Haflidason H., Hajdas I., Hatté C., Heaton T.J., Hoffman D.L., Hogg A.G., Hughen K.A., Kaiser K.F., Kromer B., Manning S.W., Niu M., Reimer R.W., Richards D.A., Scott E.M., Southon J.R., Staff R.A., Turney C.S.M. \& van der Plicht J. 2013. IntCal13 and Marine13 radiocarbon age calibration curves 0-50,000 years cal BP. Radiocarbon, 55 (4): 1869-1887.

Ruthsatz B. 2008. Hartpolstermoore der Hochanden NWArgentiniens als Indikatoren für Klimagradienten. Mitteilungen der Arbeitsgemeinschaft für Geobotanik Schleswig-Holstein und Hamburg, 65: 209-238.
Schittek K., Forbriger M., Mächtle B., Schäbitz F., Wennrich V., Reindel M. \& Eitel B. 2015. Holocene environmental changes in the highlands of the southern Peruvian Andes $\left(14^{\circ} \mathrm{S}\right)$ and their impact on pre-Columbian cultures. Climate of the Past, 11(1): 27-44. DOI: $10.5194 / \mathrm{cp}-11$ 27-2015.

Schittek K., Kock S.T., Lücke A., Hense J., Ohlendorf C., Kulemeyer J.J., Lupo L.C. \& Schäbitz F. 2016. A highaltitude peatland record of environmental changes in the NW Argentine Andes $\left(24^{\circ} \mathrm{S}\right)$ over the last 2100 years. Climate of the Past, 12(5): 1165-1180. DOI: 10.5194/cp12-1165-2016.

Squeo F.A., Wagner B.G., Aravena R. \& Espinoza D. 2006. Bofedales : high altitude peatlands of the central Andes, 79(2): 245-255. DOI:10.4067S0716078X2006000200010.

Thompson L. 1995. Late holocene ice core records of climate and environment from the tropical Andes, Peru. Bulletin de L'Institut Francais d'Etudes Andines, 24: 619-629.

Thompson L.G., Davis M.E. \& Mosley-Thompson E. 1994. Glacial records of Global climate: A 1500-year tropical ice core record of climate. Human Ecology, 22(1): 83-95. DOI: $10.1007 / \mathrm{BF} 02168764$.

Waddington J.M., Rochefort L. \& Campeau S. 2003. Sphagnum production and decomposition in a restored cutover peatland, Wetlands Ecology and Management, 11(1-2): 85-95. DOI: 10.1023/A:1022009621693.

Zhou J. \& Lau K.M. 1998. Does a monsoon climate exist over South America? Journal of Climate, 11(5): 1020-1040. DOI: $10.1175 / 1520$ 0442(1998)011<1020:DAMCEO>2.0.CO;2.

Tabla 1. Edades de radiocarbono del testigo APA01. Para las primeras cuatro fechas, se utilizó CALIBomb. El representa los valores $2 \sigma$, y la edad mediana está entre paréntesis.

\begin{tabular}{lccccll}
\hline Código & $\begin{array}{c}\text { Profundidad } \\
(\mathrm{cm})\end{array}$ & Material & Delta C13 & pMC $^{*}$ & Edad C14 & $\begin{array}{c}\text { 2 } \sigma \text { edad calibrada AP } \\
\text { (CALIBomb/Calib7.10) }\end{array}$ \\
\hline SacA43147 & $2-3$ & Turba & -29.7 & $106.913 \pm 0.328$ & Posterior a 1950 & 2003 \\
SacA43149 & $14-15$ & Turba & -31.9 & $136.971 \pm 0.423$ & Posterior a 1950 & 1976 \\
SacA43148 & $14-15$ & Planta & -32.6 & $104.549 \pm 0.322$ & Posterior a 1950 & 1957 \\
SacA43150 & $25.5-27$ & Turba & -26.9 & $102.869 \pm 0.311$ & Posterior a 1950 & 1957 \\
SacA43151 & $32.5-33$ & Turba & -28.9 & $98.585 \pm 0.31$ & $115 \pm 30$ & $148-(117)-12$ \\
SacA43152 & $50-51$ & Turba & -27.1 & $95.915 \pm 0.311$ & $335 \pm 30$ & $476-(390)-309$ \\
SacA43153 & $62-63$ & Turba & -30.6 & $87.437 \pm 0.29$ & $1080 \pm 30$ & $1013-(985)-932$ \\
SacA43154 & $79-80$ & Turba & -27.5 & $78.737 \pm 0.267$ & $1920 \pm 30$ & $1947-(1868)-1812$ \\
SacA43155 & $95-96$ & Turba & -25.8 & $74.306 \pm 0.267$ & $2385 \pm 30$ & $2492-(2410)-2345$ \\
\hline
\end{tabular}

Tabla 2. Valores máximos y mínimos de la densidad, porcentaje de carbono orgánico total (COT), tasa de sedimentación, y la tasa de acumulación de carbono (TAC) de las 2 unidades sedimentarias para el testigo APA01.

\begin{tabular}{|c|c|c|c|c|c|c|c|}
\hline Testigo & $\begin{array}{l}\text { Unidad/ } \\
\text { profundidad }\end{array}$ & Edad (AC/DC) & & $\begin{array}{c}\text { Densidad } \\
\left({\left.\mathrm{g} . \mathrm{cm}^{-3}\right)}^{-3}\right.\end{array}$ & $\begin{array}{c}\mathrm{COT} \\
(\%)\end{array}$ & $\begin{array}{c}\text { Tasa de } \\
\text { sedimentación } \\
\left(\mathrm{cm} \cdot \mathrm{añono}^{-1}\right)\end{array}$ & $\begin{array}{c}\text { TAC } \\
\left(\mathrm{g} \cdot \mathrm{m}^{-2} \cdot \mathrm{añ}^{-1}\right)\end{array}$ \\
\hline \multirow{4}{*}{ APA 01} & Unidad 1 & $2015-1795 \mathrm{DC}$ & Mínimo & 0.008 & 11 & 0.07 & 24.4 \\
\hline & $(0-38)$ & & Máximo & 0.31 & 44 & 0.41 & 173.4 \\
\hline & Unidad 2 & $1780 \mathrm{DC}-501 \mathrm{AC}$ & Mínimo & 0.009 & 23 & 0.06 & 7.2 \\
\hline & $(39-96)$ & & Máximo & 0.32 & 41 & 0.01 & 35.1 \\
\hline
\end{tabular}

AC/DC: Antes de Cristo / Después de Cristo. 

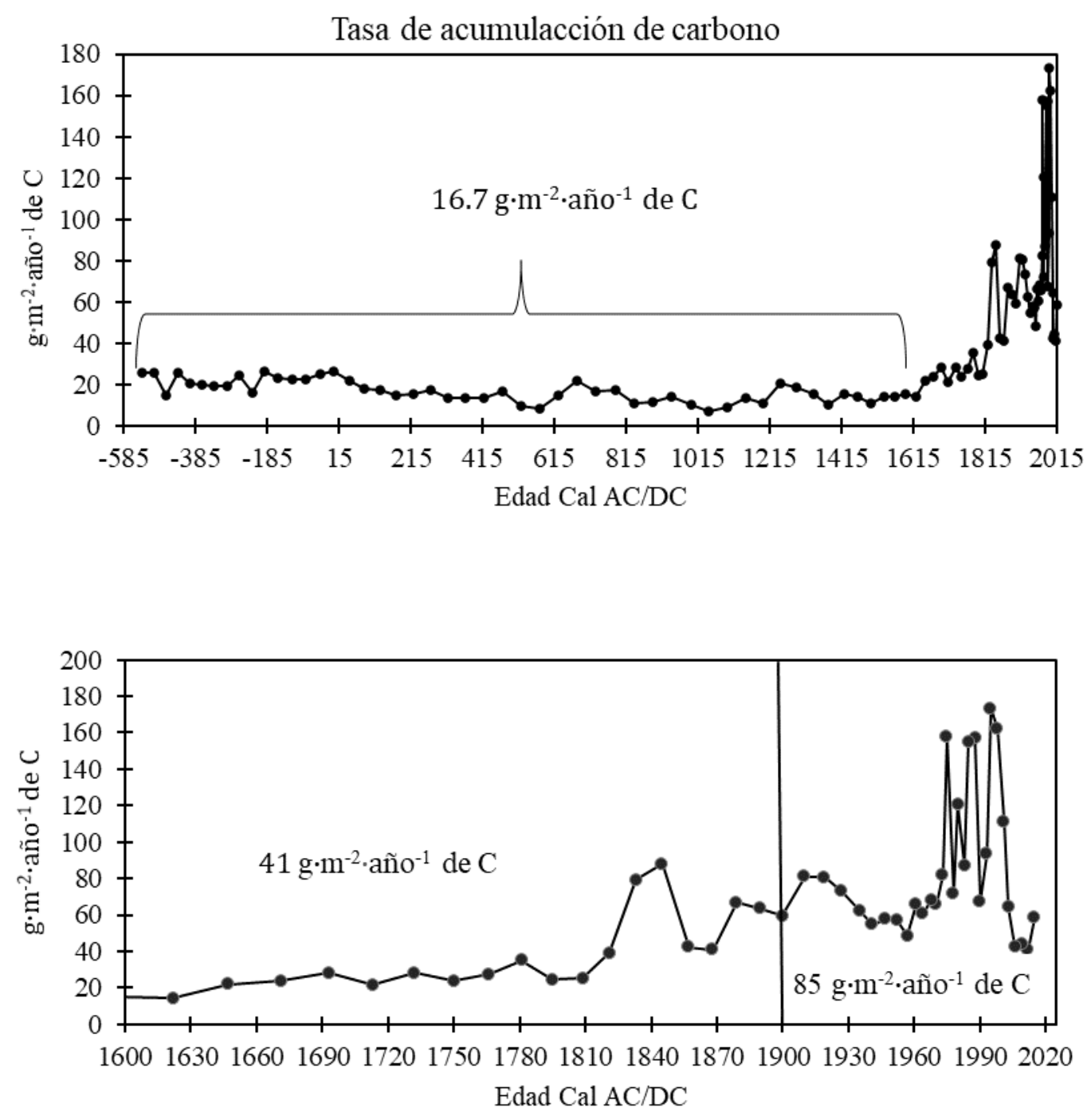

Figura 4. Tasa de acumulación de Carbono en relación edad (AC, antes de Cristo y DC, después de Cristo).

\footnotetext{
${ }^{1}$ Universidad Nacional Mayor de San Marcos, Lima / Perú; Programa Agua-Andes del Centro de Competencias del Agua, Lima / Perú. yizet.huaman@unmsm.edu.pe.

${ }^{2}$ Institut de Recherche pour le Développement (IRD)-GET (Geosciences Environnement Toulouse) UMR 5563; Universidad Nacional Agraria La Molina, Lima / Perú. patricia.turcq@ird.fr.

${ }^{3}$ Universidad Nacional Agraria la Molina, Lima / Perú; Programa Agua-Andes del Centro de Competencias del Agua, Lima / Perú. respinozavillar@gmail.com.

${ }^{4}$ Universidad Nacional Agraria la Molina, Lima / Perú; Programa Agua-Andes del Centro de Competencias del Agua, Lima / Perú. rominallanoslopez@gmail.com.

${ }^{5}$ Instituto Geofísico del Perú (IGP), Lima / Perú. japaestegui@igp.gob.pe.

${ }^{6}$ Institut de Recherche pour le Développement (LOCEAN-IPSL); Universidad Peruana Cayetano Heredia, Lima / Perú. bruno.turcq@ird.fr.

${ }^{7}$ Centro de Competencias del Agua (CCA), Lima / Perú. bwillems@cca.org.pe.
} 